Review

\title{
A Scoping Review of Climate Change, Climate-Related Disasters, and Mental Disorders among Children in Low- and Middle-Income Countries
}

\author{
Isobel Sharpe (D) and Colleen M. Davison*(D) \\ Department of Public Health Sciences, Queen's University, Kingston, ON K7L 3N6, Canada; \\ isobel.sharpe@queensu.ca \\ * Correspondence: davisonc@queensu.ca
}

check for

updates

Citation: Sharpe, I.; Davison, C.M. A Scoping Review of Climate Change, Climate-Related Disasters, and Mental Disorders among Children in Low- and Middle-Income Countries. Int. J. Environ. Res. Public Health 2022, 19, 2896. https://doi.org/10.3390/ ijerph19052896

Academic Editors: Eduardo Fonseca-Pedrero, Martin Debbané and Lawrence Palinkas

Received: 22 December 2021 Accepted: 24 February 2022 Published: 2 March 2022

Publisher's Note: MDPI stays neutral with regard to jurisdictional claims in published maps and institutional affiliations.

Copyright: (c) 2022 by the authors. Licensee MDPI, Basel, Switzerland. This article is an open access article distributed under the terms and conditions of the Creative Commons Attribution (CC BY) license (https:// creativecommons.org/licenses/by/ $4.0 /)$.

\begin{abstract}
Children, particularly those living in low- and middle-income countries (LMICs), are highly vulnerable to climate change and its impacts. Our main objective was to conduct a scoping literature review to determine how exposure to climate change and climate-related disasters influences the presence of mental disorders among children in LMICs. We also aimed to identify gaps in this area of scholarship. We included studies of children in LMICs that had a climate change or climate-related disaster exposure and mental disorder outcome. Twenty-three studies were included in the final synthesis. Fourteen studies were conducted in China, three in India, two each in Pakistan and the Philippines, and one each in Namibia and Dominica. All studies assessed the association between a climate-related disaster exposure and a mental disorder outcome, while none explored broader climate change-related exposures. Post-traumatic stress disorder ( $n=21$ studies) and depression ( $n=8$ studies) were the most common mental disorder outcomes. There was considerable betweenstudy heterogeneity in terms of sample size, follow-up length, and outcome measurement. Overall, the literature in this area was sparse. Additional high-quality research is required to better understand the impacts of climate-related disasters and climate change on mental disorders within this population to ultimately inform future policies and interventions.
\end{abstract}

Keywords: scoping review; climate change; climate-related disasters; mental health; mental disorders; children; low and middle income; LMICs

\section{Introduction}

Our climate is currently experiencing a rapid and unsustainable increase in temperature, largely driven by human greenhouse gas emissions. As of 2020, the past six years have been the warmest on record [1]. Global surface temperature has risen approximately $1.2{ }^{\circ} \mathrm{C}$ above pre-industrial levels [1], and this increase may reach up to $2{ }^{\circ} \mathrm{C}$ by the end of this century [2]. As a result, climate change has been recognized as "the biggest global health threat of the 21st century" [3]. The changing climate has a wide range of impacts on human health, such as increasing transmission of infectious diseases, increasing mortality due to heatwaves and air pollution, as well as contributing to undernutrition driven by reduced crop yields [4].

Climate change also harms human mental health and well-being [5,6]. For one, experiences of climate-related disasters, which are increasing in frequency and severity due to climate change [2], contribute to psychological trauma. For instance, exposure to flooding has been associated with a variety of negative mental health outcomes such as post-traumatic stress disorder (PTSD), depression, anxiety, and overall poor well-being [7]. Broader climate change-related events, such as biodiversity shifts and gradual temperature and sea level increase, also contribute to mental health problems. For example, rising sea levels may lead to forced migration, which contributes to mental health problems associated with overcrowding, poverty, and loss of a sense of place [8,9]. 
Individuals living in low- and middle-income countries (LMICs) are susceptible to the negative mental health effects of climate change and climate-related disasters. Like the rest of the world, many of these countries are experiencing unfavorable changes in temperature and precipitation patterns, as well as greater frequency and severity of climate-related natural disasters $[2,10]$. Due to potentially weaker health care systems and more limited financial resources for adequate infrastructure, LMICs may have less capacity for mitigation and adaptation than higher-resource areas $[2,11]$. In addition, there may be a greater need for mental health support [12,13]. Although individuals and communities will respond to climate-related adversities in their own unique ways, meaning that we cannot assume uniformity across populations, aspects of a broader context can place specific populations at potentially greater risk for negative mental health impacts. For example, LMICs where health systems are not currently providing comprehensive mental health services, or where populations are living in poverty, insecurity, or conflict may be at an overall greater risk for negative mental health outcomes. Several systematic and scoping reviews have identified LMIC-specific evidence supporting the various negative mental health outcomes associated with climate change and climate-related disasters [6,14-16].

Children are a specific subpopulation that is affected by climate change and climaterelated disasters [17]. Relative to adults, children can be more susceptible to negative environmental changes [17-19]. Evidence also suggests that stressful early-life events are frequently associated with poor health- and mental health-related outcomes later in life [20-23]. Furthermore, throughout their lifetimes, children will be forced to bear the future consequences of our widespread climate change inaction and will be required to adapt and develop solutions to these problems. For instance, based on the current climatic trajectory, Thiery et al. [24] found that children born in 2020 will experience 7-fold more heatwaves than children born in 1960. To better quantify these inequities, UNICEF [17] developed a country-level climate change risk index. The index accounted for children's level of exposure to climate change-related hazards as well as their vulnerabilities related to essential services such as water and sanitation, education, and nutrition, which often compound to produce an array of negative outcomes. According to the index, approximately half the number of children worldwide live in countries at a level of extremely high risk.

In comparison to adults, relatively little research exists on the mental health effects of climate change and climate-related disasters on children. Some narrative reviews have explored this topic [19,25-27]; however, none have captured the full extent of the existing literature. In their recent scoping review of climate change and child health, Helldén and colleagues [28] analyzed 371 records, only $12 \%$ of which focused on LMICs and an even smaller proportion on mental health. Systematically capturing relevant literature on climate change, climate-related disasters, and mental health in this context will help to identify research gaps and may eventually inform LMIC- and child-specific adaptation policies and mental health interventions.

In 2010, the Global Burden of Disease study found that mental, neurological, and substance abuse disorders accounted for over $10 \%$ of all disability-adjusted life years [29]. Mental disorders are an especially burdensome aspect of mental health, and assessing their presence is highly relevant within the context of climate change and climate-related disasters. Therefore, our main objective was to conduct a scoping literature review to determine how exposure to climate change and climate-related disasters influences the presence of mental disorders among children in LMICs. We also aimed to identify and summarize existing gaps in this area of literature.

\section{Materials and Methods}

We used the scoping review methodology to answer our research question. Scoping reviews are used to systematically map a heterogeneous body of literature [30], and we felt it was appropriate given that the settings and designs of studies investigating climate change, climate-related disasters, and mental disorders tend to vary widely. This review aligned with the Preferred Reporting Items for Systematic reviews and Meta-Analyses 
extension for Scoping Reviews (PRISMA-ScR) checklist [31] and we followed the same methodological approach as our previous scoping review, which investigated climate change and mental health among adults in LMICs [16]. The review was based on an unpublished protocol.

\subsection{Eligibility Criteria}

We included all primary research published in 2007 or later that explored the relationship between a climate change or climate-related disaster exposure and a mental disorder outcome in LMICs (Table 1). Studies were included if they focused on children, defined as having a study population with a mean age of 18 years or less. This definition corresponded with our previous work on adult-focused populations [16]; we felt it was important to capture studies that primarily included children but also those that may have included a small proportion of adults (given that this situation was common within our area of literature). We included studies with exposures that were either climate-related natural disasters or were explicitly mentioned by the study author as "climate change". The mental disorder outcomes of interest were anxiety, depression, post-traumatic stress, acute stress, substance use and addiction, bipolar, schizophrenia, suicidal behavior, and non-suicidal self-injury [32]. We included studies that evaluated mental disorders based on Diagnostic and Statistical Manual of Mental Disorders (DSM) or International Classification of Diseases (ICD) criteria. For feasibility purposes, only research written in the English language was included.

Table 1. Scoping review inclusion and exclusion criteria.

\begin{tabular}{|c|c|c|}
\hline & Inclusion Criteria & Exclusion Criteria \\
\hline Population & $\begin{array}{l}\text { - Child focused (study population with mean age } \\
18 \text { years and under) } \\
\text { - Study population located entirely in low- and } \\
\text { middle-income countries at time of exposure (may be } \\
\text { located in more than one) }{ }^{1}\end{array}$ & $\begin{array}{l}\text { - Adult focused (study population with mean } \\
\text { age over } 18 \text { years) } \\
\text { - Any part of the study population located in } \\
\text { high-income countries }\end{array}$ \\
\hline Intervention (Exposure) & $\begin{array}{l}\text { - Climate-related disaster exposure (see Table S1 for } \\
\text { list of climate-related disasters) OR climate } \\
\text { change-related exposure (as identified by the } \\
\text { study author) }{ }^{2}\end{array}$ & \\
\hline Comparison & - Any & \\
\hline Outcome & $\begin{array}{l}\text { - Mental disorders (anxiety, depression, post-traumatic } \\
\text { stress, acute stress, substance use and addiction, } \\
\text { bipolar, schizophrenia, suicidal behavior, non-suicidal } \\
\text { self-injury) evaluated based on DSM or ICD symptoms }\end{array}$ & $\begin{array}{l}\text { - Mental disorders not evaluated based on } \\
\text { DSM or ICD symptoms } \\
\text { - Study only measures positive mental health }\end{array}$ \\
\hline Study Design & $\begin{array}{l}\text { Any type of empirical literature, including } \\
\text { - Journal articles (quantitative, qualitative, or mixed } \\
\text { methods) } \\
\text { - Grey literature (conference proceedings, dissertations, } \\
\text { government and organization documents, } \\
\text { policy briefs) }\end{array}$ & $\begin{array}{l}\text { - Narrative reviews, syntheses (scoping } \\
\text { reviews, systematic reviews, meta-analyses, } \\
\text { etc.), commentaries, editorials, expert } \\
\text { opinions } \\
\text { - Validation studies } \\
\text { - Non-English language } \\
\text { - Date of publication before } 2007^{3}\end{array}$ \\
\hline
\end{tabular}

Abbreviations: Diagnostic and Statistical Manual of Mental Disorders (DSM); International Classification of Diseases (ICD). ${ }^{1}$ Based on the World Bank's designation of a low- and middle-income country (includes lowlower-middle, and upper-middle-income economies) [33]. ${ }^{2}$ Climate change is a challenging concept to define and measure. We chose to include studies where the study author explicitly mentioned that they were investigating the effects of climate change (e.g., used the words "climate change" when describing the study exposure). ${ }^{3}$ The Intergovernmental Panel on Climate Change's fourth assessment report (AR4) was published in 2007, in which the authors began to acknowledge the impacts of climate change on human health and well-being. This approach was taken in a similar scoping review by Middleton and colleagues [34]. 


\subsection{Information Sources and Search Strategy}

We conducted a comprehensive search of both the academic and grey literature. All searches were set to identify records published between 1 January 2007 and 31 December 2019. In terms of the academic literature, we searched five relevant electronic databases: MEDLINE via Ovid (biomedicine, 1946-present), Embase via Ovid (biomedicine and pharmacology, 1947-present), APA PsycInfo via Ovid (psychological, social, behavioral, and health sciences, 1806-present), Global Health via Ovid (global health, 1910-present), and Sociological Abstracts via ProQuest (sociology, 1952-present). With guidance from a health sciences librarian, the search strategy was developed in MEDLINE and translated to the four other databases (Tables S2-S6). We also performed manual searches of the 2019 volumes of the following journals to capture any studies that were not yet indexed in the electronic databases: International Journal of Environmental Research and Public Health, Global Environmental Change, Climatic Change, Disaster Medicine and Public Health Preparedness, and Environmental Health. In addition, we searched the reference lists of all included studies (backward snowballing). We also checked Google Scholar for any relevant citations of the studies (forward snowballing).

In terms of the grey literature, our search strategy was adapted from Godin et al. [35]. First, a list of relevant organizations was generated and targeted website browsing/searching was performed (Table S7). The list was generated through the authors' personal knowledge, as well as through Google searching of relevant keywords. If the website contained a search feature, it was used in combination with general browsing. Second, we searched the following grey literature databases: Google Scholar, OAIster, OpenGrey, The Grey Literature Report, ELDIS, BIOSIS Previews, Center for Research Libraries, Networked Digital Library of Theses and Dissertations, and ProQuest Dissertations \& Theses Global (Table S8). Lastly, the popular search engines Google and DuckDuckGo were searched (Table S9). The first 100 results of each search were screened.

\subsection{Screening}

First, all search results were compiled and duplicates were removed. For feasibility purposes, a single reviewer performed an initial relevancy screen of all unique records to identify those titles/abstracts clearly unrelated to the study objective (i.e., did not have an exposure related to the environment, did not have an outcome related to mental health, did not take place in a LMIC, or were not primary research). Following the initial screen, two independent reviewers completed a formal assessment of eligibility (Table 1) at the title/abstract level and any irrelevant records were excluded. The remaining records were then assessed by two reviewers at the full-text level to confirm eligibility (Table 1). Any conflicts were discussed between the two reviewers and mutual agreement was reached. All aspects of the study organization and screening were managed using the Covidence systematic review software (https:/ / app.covidence.org/, accessed on 21 December 2020 Veritas Health Innovation, Melbourne, Australia).

\subsection{Data Collection and Analysis}

Data extraction was completed for all included studies. Prior to beginning the extraction process, a comprehensive Microsoft Excel data extraction form was developed, which was piloted on a random sample of five included studies and adjusted accordingly. The form consisted of information on study design, baseline characteristics, and qualitative and quantitative study findings (Table S10). The final results were presented qualitatively in various descriptions, tables, frequency counts, and diagrams.

\section{Results}

The academic literature search returned 6971 unique records, 4536 of which were excluded through the initial relevancy screen (Figure 1). A total of 2435 records were screened for eligibility by two independent reviewers at the title/abstract level and 2076 were excluded. The remaining 359 were assessed at the full-text level and 338 were excluded. 
In combination with two additional records identified through backwards and forwards snowballing, 23 studies were included in the final qualitative synthesis. No additional records were identified through the grey literature search.

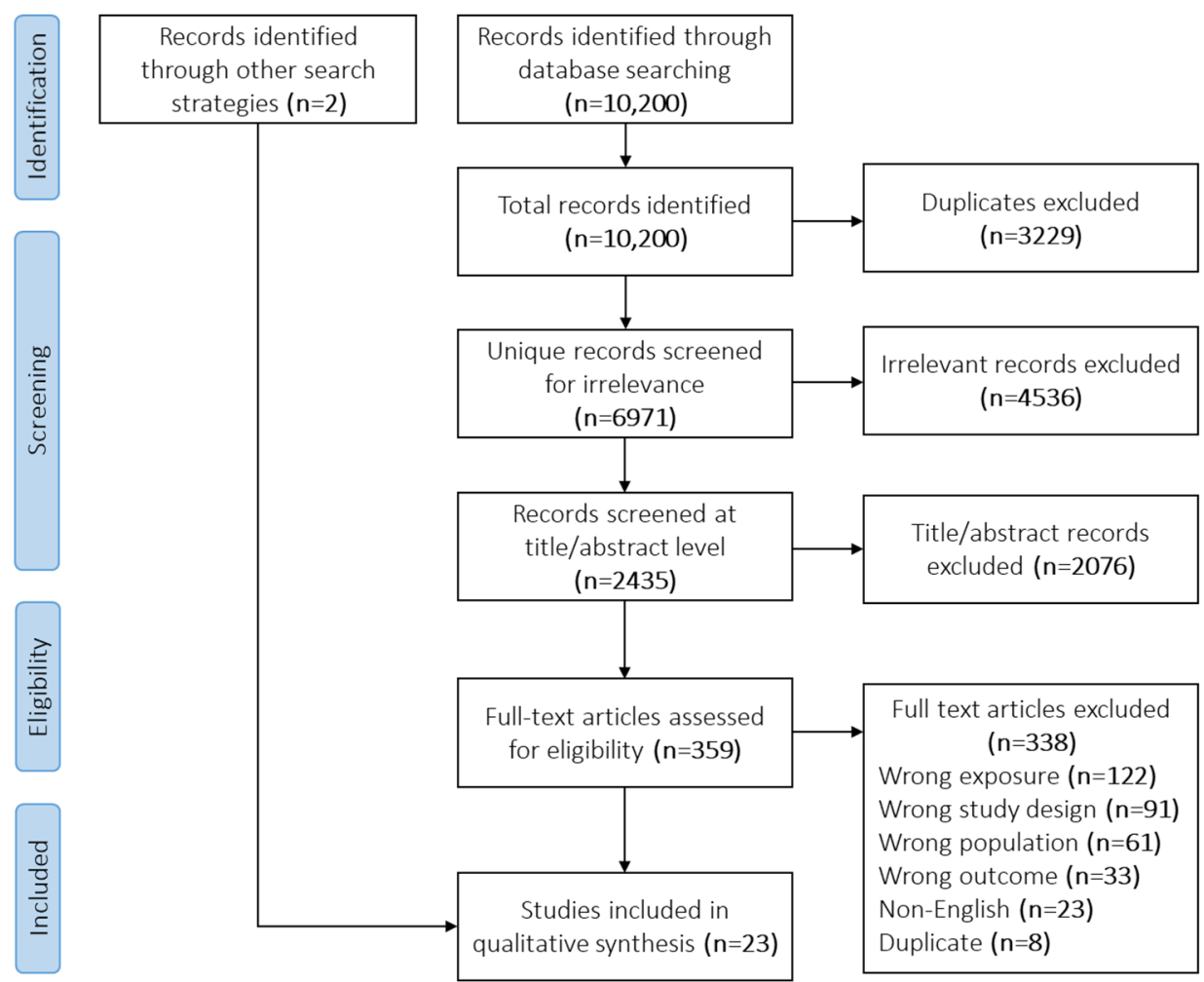

Figure 1. PRISMA diagram showing the study inclusion process.

Of the 23 included studies, 22 were quantitative only and one was mixed methods. Twenty-one studies used a cross-sectional design and the remaining two used a prospective cohort design. The included studies spanned six different LMICs: China ( $n=14$ studies), India $(n=3)$, Pakistan $(n=2)$, Philippines $(n=2)$, Namibia $(n=1)$, and Dominica $(n=1)$. All 23 studies assessed climate-related disaster exposures, while none assessed broader climate change-related exposures. Twelve unique disaster events were identified, including four floods, four typhoons/cyclones/tropical storms, two snowstorms, one rainstorm, and one tornado (Figure 2). 


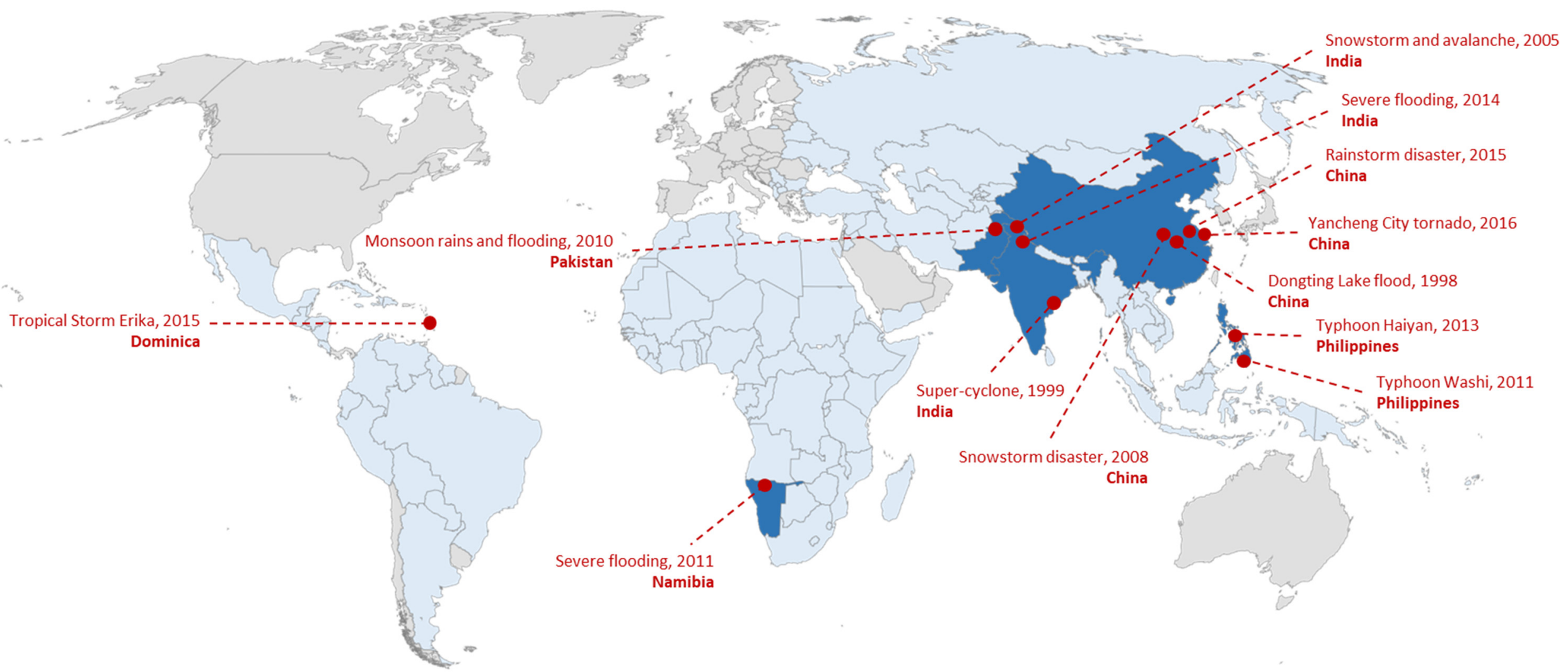

Figure 2. World map showing the 12 unique climate change exposures captured by the included studies (dark blue countries). The climate change events took place in China $(n=4)$, India $(n=3)$, Philippines $(n=2)$, Pakistan $(n=1)$, Namibia $(n=1)$, and Dominica $(n=1)$. The remaining low- and middle-income countries are colored light blue and high-income countries are colored grey. 
The most common mental disorder outcome was PTSD, assessed by 21 studies. Depression was assessed by eight studies, anxiety by two studies, and acute stress disorder by one study. One study assessed all DSM disorders. Prevalence estimates ranged from $2 \%$ [36,37] to $83 \%$ [38] for PTSD and from $2 \%$ [36] to $66 \%$ [39] for depression. While some studies assessed mental disorders using formal diagnostic interviews, e.g., [36,40,41], others used screening tools with validated cut-off scores to establish an informal diagnosis, e.g., $[38,39,42]$. The most commonly used screening tools were the Child PTSD Symptom Scale ( $n=8$ studies), the Depression Self-Rating Scale for Children $(n=4)$, and the PTSD Checklist for DSM-5 $(n=3)$.

Study sample sizes ranged from $n=64$ [43] to $n=7038$ [37]. Most $(70 \%, n=16)$ studies had a sample size between 100 and 500. Nineteen studies collected samples from schoolbased populations and four studies collected samples from community-based populations (one sampled females only). Follow-up length ranged from one week [44] to five years [40] post-exposure and the most common follow-up length was 12 months ( $n=5$ studies). Date of publication ranged from 2007-2019, with the large majority of studies $(78 \%, n=18)$ published in 2015 or later (Figure 3). Eleven studies were published in 2018 alone, eight of which presented identical or very similar samples with varying lengths of follow-up. All but one of the included studies [45] had partial or full LMIC authorship. The following sections present relevant study findings by country and Table 2 provides a detailed summary of all included studies.

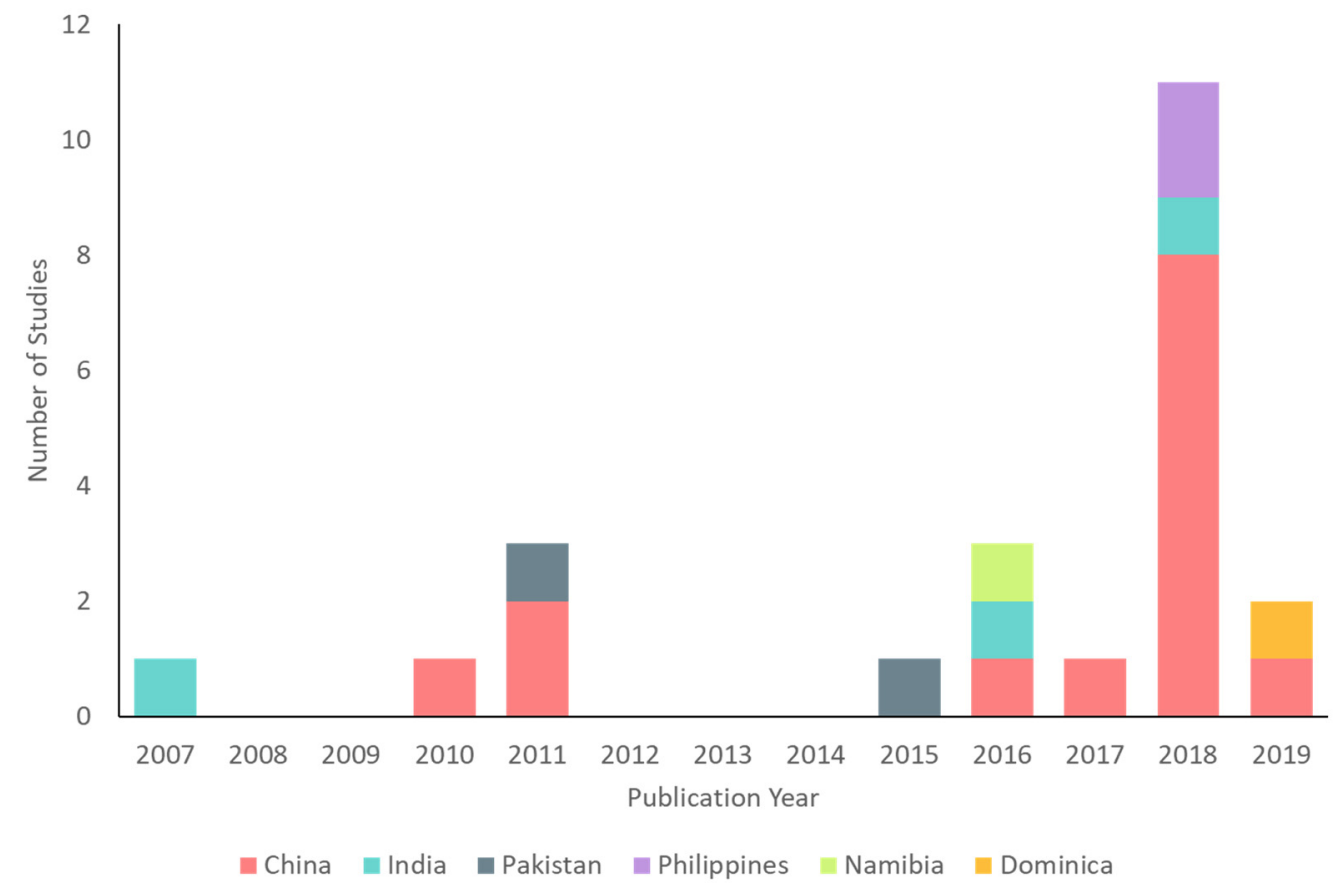

Figure 3. Included studies $(n=23)$ by year and country. 
Table 2. Summary of all included studies $(n=23)$. All studies were quantitative unless otherwise specified.

\begin{tabular}{|c|c|c|c|c|c|c|c|c|c|}
\hline Study ID & Design & $\begin{array}{c}\text { Sample Size } \\
\text { and Population }\end{array}$ & $\begin{array}{l}\text { Sampling } \\
\text { Method }\end{array}$ & $\begin{array}{l}\text { Climate-Related } \\
\text { Disaster } \\
\text { Exposure(s) }\end{array}$ & $\begin{array}{c}\text { Mental } \\
\text { Disorder } \\
\text { Outcome(s) }\end{array}$ & $\begin{array}{c}\text { Outcome } \\
\text { Measurement } \\
\text { Tool(s) }\end{array}$ & $\begin{array}{l}\text { Post-Exposure } \\
\text { Follow-Up Length }\end{array}$ & $\begin{array}{l}\text { Main Findings (on the Association } \\
\text { between Climate-Related Disasters and } \\
\text { Mental Disorders) }\end{array}$ & $\begin{array}{l}\text { Mental Disorder Prevalence } \\
\text { Estimates }\end{array}$ \\
\hline \multicolumn{10}{|c|}{ China $(n=14)$} \\
\hline $\begin{array}{c}\text { An-2019 } \\
\text { (same baseline } \\
\text { sample as Xu 2018a) } \\
\text { [39] }\end{array}$ & $\begin{array}{l}\text { Prospective } \\
\text { cohort }\end{array}$ & $\begin{array}{c}154 \text { middle } \\
\text { school students }\end{array}$ & $\begin{array}{l}\text { Multistage } \\
\text { cluster random } \\
\text { sampling }\end{array}$ & Tornado & $\begin{array}{c}\text { PTSD, } \\
\text { depression }\end{array}$ & $\begin{array}{l}\text { CPSS, CES-DC } \\
\text { (self-reported } \\
\text { surveys) }\end{array}$ & $\begin{array}{l}6 \text { (T1), } 9 \text { (T2), and } 12 \\
\text { (T3) months }\end{array}$ & & $\begin{array}{c}\text { Post-tornado PTSD and } \\
\text { depression: } 55.84 \% \text { and } \\
56.49 \% \text { at T1, 50.0\% and } \\
65.58 \% \text { at T2, } 47.40 \% \text { and } \\
66.01 \% \text { at T3; PTSD at T1 } \\
\text { significantly predicted } \\
\text { depression at T2 }(p<0.001) \\
\text { and PTSD at T2 significantly } \\
\text { predicted depression at T3 }\end{array}$ \\
\hline $\begin{array}{c}\text { An-2018a } \\
\text { (sample related } \\
\text { to Xu-2018a) } \\
{[46]}\end{array}$ & $\begin{array}{c}\text { Cross- } \\
\text { sectional }\end{array}$ & $\begin{array}{l}443 \text { junior high } \\
\text { school students }\end{array}$ & $\begin{array}{l}\text { Multistage } \\
\text { cluster random } \\
\text { sampling }\end{array}$ & Tornado & PTSD & $\begin{array}{l}\text { CPSS } \\
\text { (self-reported } \\
\text { survey) }\end{array}$ & 12 months & & \\
\hline $\begin{array}{c}\text { An-2018b } \\
\text { (same baseline } \\
\text { sample as Xu } \\
\text { 2018a) [47] }\end{array}$ & $\begin{array}{l}\text { Prospective } \\
\text { cohort }\end{array}$ & $\begin{array}{c}204 \text { middle } \\
\text { school students }\end{array}$ & $\begin{array}{l}\text { Multistage } \\
\text { cluster random } \\
\text { sampling }\end{array}$ & Tornado & PTSD & $\begin{array}{l}\text { CPSS } \\
\text { (self-reported } \\
\text { survey) }\end{array}$ & 6 and 9 months & & \\
\hline $\mathrm{Li}-2010[48]$ & $\begin{array}{c}\text { Cross- } \\
\text { sectional }\end{array}$ & $\begin{array}{l}4327 \text { children } \\
\text { aged } 7-15 \text { and } \\
\text { their parents }\end{array}$ & $\begin{array}{l}\text { Multistage } \\
\text { cluster random } \\
\text { sampling }\end{array}$ & $\begin{array}{l}\text { Flood (Dongting } \\
\text { Lake) }\end{array}$ & PTSD & $\begin{array}{l}\text { DSM-IV criteria } \\
\text { (interview) }\end{array}$ & 18 months & $\begin{array}{l}\text { Presence of PTSD was significantly } \\
\text { greater among children who experienced } \\
\text { flash or drainage problem flooding, } \\
\text { experienced moderate ( } 25-49 \% \text { of total } \\
\text { village area) or severe ( } \geq 50 \% \text { of total } \\
\text { village area) flooding, were dropped into } \\
\text { water, were trapped in water, had a } \\
\text { serious injury, had seriously injured } \\
\text { relatives, witnessed somebody drown, } \\
\text { had death of a family member or friend, } \\
\text { were trapped in water near a dead body, } \\
\text { had previous flood experience, were } \\
\text { separated from family members, had } \\
\text { teachers or classmates drown, had class } \\
\text { suspended, had the following school } \\
\text { semester postroned, and had parents } \\
\text { with PTSD (all } p \leq 0.001) ; \text { multivariate } \\
\text { analyses showed that having a PTSD } \\
\text { positive father (OR 3.0, } 95 \% \text { CI 2.05-4.50) } \\
\text { or mother (OR 4.4, 95\% CI 2.99-6.45) } \\
\text { significantly increased risk of child PTSD }\end{array}$ & $\begin{array}{l}\text { Post-flood PTSD: children } \\
4.7 \% \text {, parents } 11.2 \%\end{array}$ \\
\hline
\end{tabular}


Table 2. Cont.

\begin{tabular}{|c|c|c|c|c|c|c|c|c|c|}
\hline Study ID & Design & $\begin{array}{c}\text { Sample Size } \\
\text { and Population }\end{array}$ & $\begin{array}{l}\text { Sampling } \\
\text { Method }\end{array}$ & $\begin{array}{l}\text { Climate-Related } \\
\text { Disaster } \\
\text { Exposure(s) }\end{array}$ & $\begin{array}{c}\text { Mental } \\
\text { Disorder } \\
\text { Outcome(s) }\end{array}$ & $\begin{array}{c}\text { Outcome } \\
\text { Measurement } \\
\text { Tool(s) }\end{array}$ & $\begin{array}{l}\text { Post-Exposure } \\
\text { Follow-Up } \\
\text { Length }\end{array}$ & $\begin{array}{l}\text { Main Findings (on the Association } \\
\text { between Climate-Related Disasters } \\
\text { and Mental Disorders) }\end{array}$ & $\begin{array}{l}\text { Mental Disorder Prevalence } \\
\text { Estimates }\end{array}$ \\
\hline Peng-2011 [37] & $\begin{array}{l}\text { Cross- } \\
\text { sectional }\end{array}$ & $\begin{array}{l}7038 \text { children } \\
\text { aged } 7-15\end{array}$ & $\begin{array}{l}\text { Multistage } \\
\text { cluster random } \\
\text { sampling }\end{array}$ & $\begin{array}{l}\text { Flood (Dongting } \\
\text { Lake) }\end{array}$ & PTSD & $\begin{array}{l}\text { DSM-IV criteria } \\
\text { (interview) }\end{array}$ & $\sim 18$ months & $\begin{array}{c}\text { Flood type (flash }>\text { collapsed }>\text { soaked) } \\
\text { and whether school reopening was } \\
\text { delayed (yes }>\text { no) were significantly } \\
\text { associated with PTSD }(p<0.001)\end{array}$ & Post-flood PTSD: $2.05 \%$ \\
\hline Quan-2017 [44] & $\begin{array}{l}\text { Cross- } \\
\text { sectional }\end{array}$ & $\begin{array}{l}951 \text { middle } \\
\text { school students }\end{array}$ & $\begin{array}{l}\text { Multistage } \\
\text { cluster random } \\
\text { sampling }\end{array}$ & Rainstorms & PTSD & $\begin{array}{l}\text { PCL-5 } \\
\text { (self-reported } \\
\text { survey) }\end{array}$ & 1 week & $\begin{array}{l}\text { Presence of PTSD was significantly } \\
\text { correlated with rainstorm-related } \\
\text { experiences and perceived severity of } \\
\text { disaster }(p=0.01)\end{array}$ & $\begin{array}{l}\text { Post-rainstorm PTSD: } 15.2 \% \\
\text { identified as probable cases }\end{array}$ \\
\hline Xu-2018a [49] & $\begin{array}{c}\text { Cross- } \\
\text { sectional }\end{array}$ & $\begin{array}{l}247 \text { middle } \\
\text { school students } \\
\text { (grades } 7-9 \text { ) }\end{array}$ & $\begin{array}{l}\text { Multistage } \\
\text { cluster random } \\
\text { sampling }\end{array}$ & Tornado & $\begin{array}{l}\text { PTSD, } \\
\text { depression }\end{array}$ & $\begin{array}{l}\text { CPSS, CES-DC } \\
\text { (self-reported } \\
\text { surveys) }\end{array}$ & 3 months & $\begin{array}{c}\text { Significantly greater odds of PTSD } \\
\text { among children who had injured } \\
\text { relatives/ friends (OR 1.98, } 95 \% \text { CI } \\
1.01-3.92) \text {, feared injury/death (OR } \\
1.92,95 \% \text { CI 1.14-3.24); significantly } \\
\text { greater odds of depression among } \\
\text { children who had injured } \\
\text { relatives / friends (OR 2.05, } 95 \% \text { CI } \\
\text { 1.03-4.07) }\end{array}$ & $\begin{array}{l}\text { Post-tornado PTSD: } 57.5 \% \text {, } \\
\text { depression: } 58.7 \%\end{array}$ \\
\hline $\begin{array}{c}\text { Xu-2018b } \\
\text { (sample related to } \\
\text { Xu-2018a) } \\
{[50]}\end{array}$ & $\begin{array}{l}\text { Cross- } \\
\text { sectional }\end{array}$ & $\begin{array}{l}431 \text { middle } \\
\text { school } \\
\text { students }\end{array}$ & $\begin{array}{l}\text { Multistage } \\
\text { cluster random } \\
\text { sampling }\end{array}$ & Tornado & Depression & $\begin{array}{l}\text { CES-DC } \\
\text { (self-reported } \\
\text { survey) }\end{array}$ & 9 months & & \\
\hline $\begin{array}{c}\text { Xu-2018c } \\
\text { (same sample as } \\
\text { Xu-2018a) [51] }\end{array}$ & $\begin{array}{c}\text { Cross- } \\
\text { sectional }\end{array}$ & $\begin{array}{l}247 \text { middle } \\
\text { school } \\
\text { students }\end{array}$ & $\begin{array}{l}\text { Multistage } \\
\text { cluster random } \\
\text { sampling }\end{array}$ & Tornado & $\begin{array}{l}\text { PTSD, } \\
\text { depression }\end{array}$ & $\begin{array}{l}\text { CPSS, CES-DC } \\
\text { (self-reported } \\
\text { surveys) }\end{array}$ & 6 months & & \\
\hline $\begin{array}{c}\text { Yuan-2018a } \\
\text { (sample related to } \\
\text { Xu-2018a) } \\
\text { [52] }\end{array}$ & $\begin{array}{l}\text { Cross- } \\
\text { sectional }\end{array}$ & $\begin{array}{l}431 \text { middle } \\
\text { school } \\
\text { students }\end{array}$ & $\begin{array}{l}\text { Multistage } \\
\text { cluster random } \\
\text { sampling }\end{array}$ & Tornado & PTSD & $\begin{array}{l}\text { CPSS } \\
\text { (self-reported } \\
\text { survey) }\end{array}$ & 9 months & & \\
\hline $\begin{array}{l}\text { Yuan-2018b } \\
\text { (same sample as } \\
\text { Xu-2018a) [53] }\end{array}$ & $\begin{array}{c}\text { Cross- } \\
\text { sectional }\end{array}$ & $\begin{array}{l}247 \text { middle } \\
\text { school } \\
\text { students }\end{array}$ & $\begin{array}{l}\text { Multistage } \\
\text { cluster random } \\
\text { sampling }\end{array}$ & Tornado & PTSD & $\begin{array}{l}\text { CPSS } \\
\text { (self-reported } \\
\text { survey) }\end{array}$ & 3 months & & \\
\hline
\end{tabular}


Table 2. Cont.

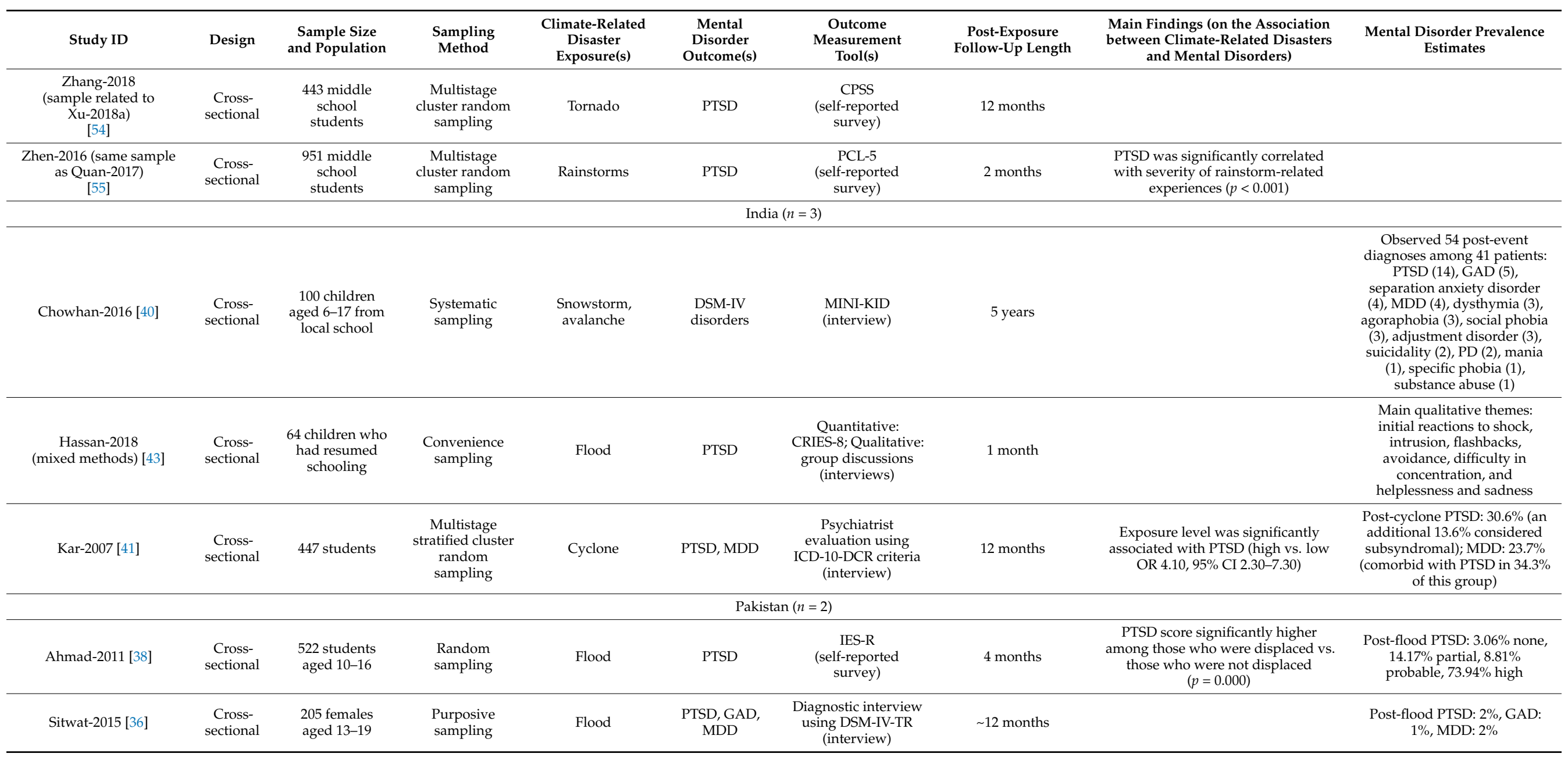


Table 2. Cont.

\begin{tabular}{|c|c|c|c|c|c|c|c|c|c|}
\hline Study ID & Design & $\begin{array}{l}\text { Sample Size } \\
\quad \text { and } \\
\text { Population }\end{array}$ & $\begin{array}{l}\text { Sampling } \\
\text { Method }\end{array}$ & $\begin{array}{l}\text { Climate- } \\
\text { Related } \\
\text { Disaster } \\
\text { Exposure(s) }\end{array}$ & $\begin{array}{c}\text { Mental } \\
\text { Disorder } \\
\text { Outcome(s) }\end{array}$ & $\begin{array}{c}\text { Outcome } \\
\text { Measurement } \\
\text { Tool(s) }\end{array}$ & $\begin{array}{l}\text { Post-Exposure } \\
\text { Follow-Up } \\
\text { Length }\end{array}$ & $\begin{array}{c}\text { Main Findings (on the } \\
\text { Association between } \\
\text { Climate-Related Disasters and } \\
\text { Mental Disorders) }\end{array}$ & $\begin{array}{l}\text { Mental Disorder } \\
\text { Prevalence Estimates }\end{array}$ \\
\hline \multicolumn{10}{|c|}{ Philippines $(n=2)$} \\
\hline Mordeno-2018 [56] & $\begin{array}{l}\text { Cross- } \\
\text { sectional }\end{array}$ & $\begin{array}{l}225 \text { child and } \\
\text { adolescent } \\
\text { survivors at } \\
\text { evacuation } \\
\text { centers }\end{array}$ & $\begin{array}{l}\text { Convenience } \\
\text { sampling }\end{array}$ & $\begin{array}{l}\text { Typhoon } \\
\text { (Washi) }\end{array}$ & $\begin{array}{c}\text { ASD, } \\
\text { depression }\end{array}$ & $\begin{array}{l}\text { ASDI (interview), } \\
\text { DSRS-C } \\
\text { (self-reported } \\
\text { survey) }\end{array}$ & 1 month & & \\
\hline \multicolumn{10}{|c|}{ Namibia $(n=1)$} \\
\hline Taukeni-2016 [58] & $\begin{array}{l}\text { Cross- } \\
\text { sectional }\end{array}$ & $\begin{array}{l}429 \text { students } \\
\text { aged 8-18 }\end{array}$ & $\begin{array}{l}\text { Stratified } \\
\text { sampling }\end{array}$ & Flood & PTSD & $\begin{array}{c}\text { CTSQ } \\
\text { (self-reported } \\
\text { survey) }\end{array}$ & 2 years & & $\begin{array}{l}\text { Post-flood PTSD: } 72.8 \% \text { of } \\
\text { children } 13+, 55.2 \% \text { of } \\
\text { children }<13\end{array}$ \\
\hline \multicolumn{10}{|c|}{ Dominica $(n=1)$} \\
\hline Tavernier-2019 [45] & $\begin{array}{l}\text { Cross- } \\
\text { sectional }\end{array}$ & $\begin{array}{l}174 \text { college } \\
\text { students }\end{array}$ & $\begin{array}{l}\text { Not specified } \\
\text { (sample from } \\
\text { a larger study) }\end{array}$ & $\begin{array}{c}\text { Tropical } \\
\text { storm (Erika) }\end{array}$ & PTSD & $\begin{array}{l}\text { Adapted PTSD } \\
\text { Checklist } \\
\text { (self-reported } \\
\text { survey) }\end{array}$ & 6 months & $\begin{array}{c}\text { PTSD was significantly } \\
\text { correlated with severity of } \\
\text { tropical storm exposure }(p<0.05)\end{array}$ & \\
\hline & & $\begin{array}{l}\text { Abbreviations: As } \\
\text { or Children), CE } \\
\text { Child Trauma Sc } \\
\text { f Events Scale- } \\
\text { DSM-5), PD (pan }\end{array}$ & $\begin{array}{l}\text { (acute stress dis } \\
\text { C (Center for } \mathrm{E} \\
\text { ening Questionn } \\
\text { evised), MDD (1 } \\
\text { disorder), and P }\end{array}$ & $\begin{array}{l}\text { der), ASDI (Act } \\
\text { demiologic Stud } \\
\text { e), GAD (gener } \\
\text { ijor depressive } \\
\text { D (post-trauma }\end{array}$ & $\begin{array}{l}\text { Stress Disorde } \\
\text { Depression Sc } \\
\text { zed anxiety dis } \\
\text { order), MINI-I } \\
\text { stress disorde }\end{array}$ & $\begin{array}{l}\text { terview), DSM (Dia } \\
\text { for Children), CPSs } \\
\text { der), ICD-DCR (Inte } \\
\text { (Mini Internation }\end{array}$ & $\begin{array}{l}\text { stic and Statistica } \\
\text { hild PTSD Sympt } \\
\text { ional Classificatic } \\
\text { Jeuropsychiatric }\end{array}$ & $\begin{array}{l}\text { Ianual of Mental Disorders), DSRS- } \\
\text { Scale), CRIES-8 (Children's Revise } \\
\text { of Diseases-Diagnostic Classificati } \\
\text { erview for children and adolescen }\end{array}$ & $\begin{array}{l}\text { (Depression Self-Rating Scale } \\
\text { Impact of Event Scale), CTSQ } \\
\text { a for Research), IES-R (Impact } \\
\text { ), PCL-5 (PTSD Checklist for }\end{array}$ \\
\hline
\end{tabular}




\subsection{China}

Fourteen studies were conducted in China. Of those studies, 11 investigated the same disaster event: a 2016 tornado in Yancheng City. Xu et al. [49] measured post-tornado PTSD and depression among a sample of middle school students, reporting 3 month prevalence estimates of $57.5 \%$ and $58.7 \%$, respectively. In addition, the authors found significantly greater odds of both disorders among students with family / friends who were injured by the tornado (PTSD: odds ratio (OR) 1.98, 95\% confidence interval (CI) 1.01-3.92; depression: OR 2.05, 95\% CI 1.03-4.07). A number of studies presented additional cross-sectional analyses of the same sample at three [53], six [39,47,51], nine [39,47], and 12 [39] months post-tornado, each exploring a slightly different conceptual model. In addition, several other studies appeared to use overlapping samples [46,50,52,54]. Most of these studies presented mental disorder prevalence estimates among children who were exposed to the climate-related disaster but did not compare prevalence at different levels of exposure.

Among the remaining five studies, both Li et al. [48] and Peng et al. [37] investigated PTSD among children 7-15 years of age who were exposed to the 1998 flooding of Dongting Lake, an event that affected approximately 223 million people [59]. Both studies reported significant relationships between flood-related exposure severity and the presence of PTSD. For example, Li et al. [48] found that PTSD was significantly greater among children who experienced more severe flooding, were dropped or trapped in the water, witnessed somebody drown or die, were separated from family members, or had a relative or friend die $(p \leq 0.001)$. Quan et al. [44] and Zhen et al. [55] both investigated PTSD among a sample of 951 middle school students who were exposed to a rainstorm disaster at one week and two month follow-ups, respectively. One-week post-rainstorm, the prevalence of PTSD was $15.2 \%$ [44]. Further, both studies reported significant associations between rainstorm-related experiences of disruption/loss and PTSD $(p \leq 0.01)[44,55]$. Finally, $\mathrm{Wu}$ and colleagues [42] studied 968 students who walked home during a snowstorm and reported a significant association between walking distance and PTSD (OR 1.01, 95\% CI 1.00-1.01).

\subsection{India}

India was the location of three studies. Five years after a snowstorm and avalanche event, Chowhan and colleagues [40] interviewed 100 schoolchildren using the Mini International Neuropsychiatric Interview for Children and Adolescents. Fifty-four diagnoses were reported among 41 children, the most common being PTSD (14 cases), generalized anxiety disorder (GAD; 5 cases), separation anxiety disorder (4 cases), and major depressive disorder (MDD; 4 cases). Hassan et al. [43] conducted a mixed-methods study of 64 child survivors of flooding. In group discussions one month after the flood, the three most common themes were difficulty in concentration (discussed by $n=44$ children), initial reactions to shock $(n=35)$, and flashbacks $(n=34)$. Neither of these studies compared outcomes between different levels of disaster exposure. Lastly, Kar et al. [41] studied PTSD and MDD among 447 schoolchildren who experienced a super-cyclone one year prior. Notably, children who lived in areas of higher exposure (i.e., areas submerged under water, with high levels of death and destruction) were significantly more likely to have PTSD compared to children in areas of lower exposure (OR 4.10, 95\% CI 2.30-7.30).

\subsection{Pakistan}

Two studies were conducted in Pakistan. Ahmad et al. [38] captured flood-related PTSD among 522 randomly selected students. Four months after the event, more than $80 \%$ of the sample had PTSD according to a score $\geq 33$ on the Impact of Events ScaleRevised [60]. In addition, students who were displaced by the flooding had significantly higher scores compared to those who were not displaced $(p=0.000)$. Sitwat and colleagues [36] used diagnostic interviews to identify the presence of mental disorders among 205 adolescent females approximately one year after a flood event. The prevalence of PTSD, 
GAD, and MDD was $2 \%, 1 \%$, and $2 \%$, respectively. All those who received a diagnosis had flood-related damage to their home.

\subsection{Philippines}

Two studies took place in the Philippines, both assessing the mental disorder effects of typhoon exposure. Mordeno and colleagues [56] measured acute stress disorder and depression among 225 child and adolescent survivors of Typhoon Washi, a severe tropical storm that led to extreme flooding and mudslides in the southern part of the country. The main goal of the study was to determine whether traumatic memories predicted negative mental disorder outcomes, and therefore the authors did not report mental disorder prevalence estimates. Nalipay and Moderno [57] measured PTSD among 446 Filipino college students three months after Typhoon Haiyan, which killed 6300 people [61]. Based on the PTSD Checklist for DSM-5 [62], 16\% of the sample had PTSD.

\subsection{Namibia}

Using a cross-sectional design, Taukeni et al. [58] explored signs of PTSD among 429 schoolchildren two years after severe flooding in the Oshana region. PTSD was measured using the Child Trauma Screening Questionnaire. The main purpose of the study was to evaluate whether the post-disaster support services provided to schools were helping children cope, and therefore the authors did not compare prevalence of mental disorders at different levels of flood exposure.

\subsection{Dominica}

Lastly, Tavernier et al. [45] conducted a cross-sectional study of 174 local college students from Dominica six months after exposure to Tropical Storm Erika. Storm exposure was measured using a 7-item checklist adapted from the Hurricane-Related Traumatic Experiences scale and storm-related PTSD was measured using an 11-item checklist adapted from the PTSD Checklist. Notably, the bivariate analysis showed that PTSD was significantly correlated with greater tropical storm exposure (correlation coefficient $0.43, p<0.05$ ).

\section{Discussion}

Children living in LMICs are susceptible to the mental health burdens of climate change and climate-related disasters. Through our scoping review, we identified 23 studies exploring how exposure to climate-related disasters influences the presence of mental disorders among children in LMICs. Overall, there was a limited amount of evidence supporting an association between these two variables.

All studies included in this review investigated climate-related disaster exposures such as floods and typhoons, while none investigated broader climate change-related exposures such as gradual changes in temperature and precipitation. A similar pattern was identified in Sharpe and Davison [16] among an adult-focused LMIC population, where over $90 \%$ of the included studies explored climate-related disaster exposures. These exposures are often easier to quantify; climate-related disasters tend to have objective geographical boundaries and set start and end dates. Comparatively, indirect broader climate change exposures often occur over extended periods of time and place, making them more challenging to quantify. Future research should aim to examine the influence of the many diverse impacts of climate change on child mental health.

We found that few studies in our review tested the association between climate-related disasters and mental disorders. Although all included studies had a climate-related disaster exposure and mental disorder outcome, many tested complex conceptual models that did not directly explore our association of interest (e.g., $[47,50,56])$. However, we chose to include these studies due to their relevance to the topic area; there is a dearth of childspecific literature focusing on these particular variables. Further, none of the studies compared mental disorders between groups who were exposed to a climate-related disaster and groups who were unexposed, making it challenging to understand the true association 
between these two variables. Existing reviews have also identified a lack of evidence on the association between climate change and child health in LMICs $[28,63]$. Ultimately, more well-designed studies are needed, especially those making unexposed group comparisons that allow for direct tests of the association between climate change or climate-related disasters and mental health. This being said, it is also important to acknowledge that many of the studies identified by our review took place in post-disaster and under-resourced settings, posing various challenges such as physical inaccessibility and loss of records [64]. Despite these barriers, other studies have been successful in comparing mental disorders between those exposed and unexposed to a climate-related disaster event. For example, in an adult population, Amstadter and colleagues [65] collected mental health data before and after the occurrence of Typhoon Xangsane in the Philippines, allowing for a pre-post comparison. Wind and colleagues [66] compared anxiety and depression scores between adults who were exposed to a flood event and a similar group who was not exposed. These examples provide potential study designs to be applied in child populations to gain a better understanding of the relationship between climate-related disasters and mental disorders.

Despite the lack of unexposed group comparisons, several studies assessed climaterelated disaster exposure severity (i.e., level of exposure) as a risk factor for mental disorders, generally identifying significant exposure-outcome associations [37,38,41,42,44,45,48,49,55]. A narrative review conducted by Burke et al. [25] highlighted similar findings, particularly among children living in LMICs. A number of reviews have also recognized similar trends among adult populations. For example, Rataj et al. [14] identified increased burdens of PTSD, anxiety, and depression associated with climate-related disasters in developing countries. Future research efforts should focus on expanding this evidence base for children specifically given that the biological pathways through which climate change affects mental health may be distinct among this group, potentially leading to lasting or compounded outcomes throughout the life course [20-23]. Although our findings provide some evidence supporting a relationship between climate-related disasters and increased mental disorders among children in LMICs, further child-specific information is needed.

The studies included in our review used a variety of measures to assess mental disorder outcomes. While some studies conducted formal assessments using diagnostic interviews, such as the Mini International Neuropsychiatric Interview for children and adolescents [40], others used informal screening tools, such as the Childhood Trauma Screening Questionnaire [58] or the Depression Self-Rating Scale for Children [56]. Although the validity and reliability of screening tools may vary, they play an important role in rapidly identifying mental health problems among children in post-disaster settings [67]. Further, screening tools are particularly useful in LMIC settings, where health care workers may not have the qualifications or the time to conduct formal diagnostic interviews [68].

In addition, 21 of the 23 studies included in this review used a cross-sectional design. Cross-sectional designs may lack temporality, where in some contexts it becomes challenging to identify whether the climate-related disaster exposure truly preceded the mental disorder outcome. Included in our review, An et al. [39] and An et al. [47] conducted prospective cohort studies that measured PTSD among a group of middle school students at 6,9, and 12 months after a tornado. Longitudinal approaches such as these may help to identify the changing effects of climate-related disasters on mental disorders over time, providing important evidence for optimizing the timing and duration of post-disaster childhood mental health interventions.

The patterns identified in this review largely aligned with those identified by a similar review focused on an adult LMIC population [16]. More specifically, both reviews identified some limited support for the relationship between climate-related disasters and increased mental disorders, although the evidence base is considerably smaller among children. While more research is needed to understand the child-specific impacts of the changing climate, various adaptation policies and mental health interventions are currently being researched and implemented. In disaster contexts, there is child-specific evidence supporting the effectiveness of cognitive behavioral therapy, narrative exposure, meditation relaxation, 
group therapy, and play therapy for improving mental health [67,69]. Kar et al. [67] also emphasized the need to train disaster relief workers to appropriately support children in these situations, for example through the use of emotional first aid. In the more general context of climate change, UNICEF [17] recommends investments in childhood education, health, nutrition, and poverty reduction. For example, they predict that investments in education, through creating infrastructure resilient to disasters and expanding schooling so that children are empowered to mitigate climate change, could reduce climate risk for more than 275 million children [17]. These actions will also subsequently reduce potential negative mental health impacts.

\section{Strengths and Limitations}

Our review had several strengths. First, we performed comprehensive searches of both the grey and academic literature. In conjunction with a health sciences librarian, we developed a detailed search strategy to capture literature from five relevant electronic databases (MEDLINE, Embase, APA PsycInfo, Global Health, and Sociological Abstracts). We also searched recent issues of relevant journals and performed reference snowballing for all included studies. We used a three-step strategy to search the grey literature, although it did not return any results. Similarly, Charlson et al. [6] performed a systematic search of climate change and mental health among all populations and also did not identify any relevant grey literature, suggesting that our search was rigorous. Second, our work was novel given that no previous reviews have explored this specific topic. While others have investigated climate change, climate-related disasters, and mental health among individuals in LMICs $[14,16,70]$, none have explored this topic specifically among children. Children in LMICs are susceptible to the negative mental health impacts of climate change, thus we felt it was important to capture the current state of this literature to identify gaps and determine important next steps for research.

This review also had limitations. For one, we only included studies written in the English language. This decision was made due to project feasibility; however, non-English studies making useful contributions to the research question were therefore excluded. Our search was also limited to studies published in 2007 or later. We made this decision because the Intergovernmental Panel on Climate Change had not acknowledged the effects of climate change on human health and well-being before this date. Thus, we would have missed any relevant studies published before 2007. However, Charlson et al. [6]'s systematic search was performed from 2001 onwards and did not identify any studies published before 2007, suggesting that we captured the full extent of the literature. Given the close relationship between climate change and extreme weather events [2], we created an evidence-based list of climate-related natural disasters to include in this review (Table S1). While it is reasonably likely that climate change played a role in many of these disasters, some may have occurred for unrelated reasons. This decision may have resulted in several false-positive studies; however, we felt this was justified given that the effects of climate change are challenging to define and measure. In addition, as our search focused on mental disorders, it did not explore all concepts related to mental health or well-being. Suldo and Shaffer [71] and Antaramian et al. [72] present the dual-factor model of mental health, which suggests that while mental health has often been described in relation to the presence or absence of psychopathology, it should also be represented by positive indicators such as well-being. For feasibility reasons, we limited our search to only include mental disorder outcomes. Future research should include subclinical mental illness, mental health, well-being, and resilience outcomes among children in LMICs. Lastly, we did not formally assess study quality using a risk of bias tool. Formal quality assessments are not typically performed in a scoping review [31]. Informally, we observed substantial variation in study quality. Some studies provided little to no information on sampling techniques, measurement tools, and/or statistical analysis. Nonetheless, the main objective of our review was to capture the scope of the literature, regardless of its quality. 


\section{Conclusions}

Climate change and its impacts represent a substantial threat to children, especially those living in LMICs. We used the scoping review methodology to determine how exposure to climate change and climate-related disasters influences the presence of mental disorders within this population. We found that the literature in this area was fairly sparse. Importantly, more well-designed studies on this topic are required, including those with unexposed comparison groups that avoid issues related to temporality. Improvements in the quality and quantity of research will create an evidence base to support policy and interventional efforts for reducing the negative mental health effects of climate change.

Supplementary Materials: The following supporting information can be downloaded at: https: / / www.mdpi.com/article/10.3390/ijerph19052896/s1, Table S1: Inclusion and exclusion criteria for climate-related disaster exposures; Table S2: Detailed MEDLINE search strategy; Table S3: Detailed EMBASE search strategy; Table S4: Detailed PsycInfo search strategy; Table S5: Detailed Global Health search strategy; Table S6: Detailed Sociological Abstracts search strategy; Table S7: Grey literature search strategy for targeted website browsing/searching; Table S8: Grey literature search strategy for database searching; Table S9: Grey literature search strategy for search engine searching; Table S10: Information collected by the data extraction form.

Author Contributions: Conceptualization, I.S. and C.M.D.; methodology, I.S. and C.M.D.; validation, I.S. and C.M.D.; formal analysis, I.S.; investigation, I.S.; resources, C.M.D.; data curation, I.S.; writingoriginal draft preparation, I.S.; writing - review and editing, C.M.D.; visualization, I.S.; supervision, C.M.D.; project administration, C.M.D.; funding acquisition, C.M.D. All authors have read and agreed to the published version of the manuscript.

Funding: This research was funded by the Canadian Institutes for Health Research, grant numbers PJT-162463 and PJT-165971, the Social Sciences and Humanities Research Council, grant number 435-2019-1083, PI Frank Elgar. This research was also funded by the Canadian Institutes for Health Research Fredrick Banting and Charles Best Canada Graduate Scholarship (Master's Award).

Institutional Review Board Statement: Not applicable.

Informed Consent Statement: Not applicable.

Data Availability Statement: The data presented in this study are available within this article and its supplement.

Acknowledgments: The authors would like to thank Leena AlShenaiber and Fiona Sharpe for their contributions to the study screening.

Conflicts of Interest: The authors declare no conflict of interest.

\section{References}

1. World Meteorological Organization. State of the Global Climate 2020; World Meteorological Organization: Geneva, Switzerland, 2021; p. 56.

2. Intergovernmental Panel on Climate Change. Climate Change 2014: Synthesis Report. Contribution of Working Groups I, II and III to the Fifth Assessment Report of the Intergovernmental Panel on Climate Change; Intergovernmental Panel on Climate Change: Geneva, Switzerland, 2014; p. 151.

3. Costello, A.; Abbas, M.; Allen, A.; Ball, S.; Bell, S.; Bellamy, R.; Friel, S.; Groce, N.; Johnson, A.; Kett, M.; et al. Managing the health effects of climate change: Lancet and University College London Institute for Global Health Commission. Lancet 2009, 373, 1693-1733. [CrossRef]

4. Watts, N.; Amann, M.; Arnell, N.; Ayeb-Karlsson, S.; Beagley, J.; Belesova, K.; Boykoff, M.; Byass, P.; Cai, W.; Campbell-Lendrum, D.; et al. The 2020 report of the Lancet countdown on health and climate change: Responding to converging crises. Lancet 2021, 397, 129-170. [CrossRef]

5. Berry, H.L.; Bowen, K.; Kjellstrom, T. Climate change and mental health: A causal pathways framework. Int. J. Public Health 2010, 55, 123-132. [CrossRef]

6. Charlson, F.; Ali, S.; Benmarhnia, T.; Pearl, M.; Massazza, A.; Augustinavicius, J.; Scott, J.G. Climate change and mental health: A scoping review. Int. J. Environ. Res. Public Health 2021, 18, 4486. [CrossRef] [PubMed]

7. Fernandez, A.; Black, J.; Jones, M.; Wilson, L.; Salvador-Carulla, L.; Astell-Burt, T.; Black, D. Flooding and mental health: A systematic mapping review. PLoS ONE 2015, 10, e0119929. [CrossRef] 
8. Shultz, J.M.; Rechkemmer, A.; Rai, A.; McManus, K.T. Public health and mental health implications of environmentally induced forced migration. Disaster Med. Public Health Prep. 2019, 13, 116-122. [CrossRef]

9. Cunsolo, A.; Ellis, N.R. Ecological grief as a mental health response to climate change-related loss. Nat. Clim. Chang. 2018, 8, 275-281. [CrossRef]

10. Knox, J.; Hess, T.; Daccache, A.; Wheeler, T. Climate change impacts on crop productivity in Africa and South Asia. Environ. Res. Lett. 2012, 7, 034032. [CrossRef]

11. Wirtz, A. Natural disasters and the insurance industry. In The Economic Impacts of Natural Disasters; Guha-Sapir, D., Santos, I., Borde, A., Eds.; Oxford University Press: New York, NY, USA, 2013; pp. 128-153. ISBN 978-0-19-984193-6.

12. Rathod, S.; Pinninti, N.; Irfan, M.; Gorczynski, P.; Rathod, P.; Gega, L.; Naeem, F. Mental health service provision in low- and middle-income countries. Health Serv. Insights 2017, 10, 1178632917694350. [CrossRef]

13. Saraceno, B.; van Ommeren, M.; Batniji, R.; Cohen, A.; Gureje, O.; Mahoney, J.; Sridhar, D.; Underhill, C. Barriers to improvement of mental health services in low-income and middle-income countries. Lancet 2007, 370, 1164-1174. [CrossRef]

14. Rataj, E.; Kunzweiler, K.; Garthus-Niegel, S. Extreme weather events in developing countries and related injuries and mental health disorders-A systematic review. BMC Public Health 2016, 16, 1020. [CrossRef] [PubMed]

15. Beaglehole, B.; Mulder, R.T.; Frampton, C.M.; Boden, J.M.; Newton-Howes, G.; Bell, C.J. Psychological distress and psychiatric disorder after natural disasters: Systematic review and meta-analysis. Br. J. Psychiatry 2018, 213, 716-722. [CrossRef] [PubMed]

16. Sharpe, I.; Davison, C.M. Climate change, climate-related disasters and mental disorder in low- and middle-income countries: A scoping review. BMJ Open 2021, 11, e051908. [CrossRef]

17. UNICEF. The Climate Crisis is a Child Rights Crisis: Introducing the Children's Climate Risk Index; UNICEF: New York, NY, USA, 2021; p. 128.

18. Sheffield, P.E.; Landrigan, P.J. Global climate change and children's health: Threats and strategies for prevention. Environ. Health Perspect. 2011, 119, 291-298. [CrossRef]

19. Hanna, R.; Oliva, P. Implications of climate change for children in developing countries. Future Child. 2016, 26, 115-132. [CrossRef]

20. Goodwin, R.D.; Sourander, A.; Duarte, C.S.; Niemelä, S.; Multimäki, P.; Nikolakaros, G.; Helenius, H.; Piha, J.; Kumpulainen, K.; Moilanen, I.; et al. Do mental health problems in childhood predict chronic physical conditions among males in early adulthood? Evidence from a community-based prospective study. Psychol. Med. 2009, 39, 301-311. [CrossRef]

21. Faravelli, C.; Sauro, C.L.; Godini, L.; Lelli, L.; Benni, L.; Pietrini, F.; Lazzeretti, L.; Talamba, G.A.; Fioravanti, G.; Ricca, V. Childhood stressful events, HPA axis and anxiety disorders. World J. Psychiatry 2012, 2, 13-25. [CrossRef]

22. Mersky, J.P.; Topitzes, J.; Reynolds, A.J. Impacts of adverse childhood experiences on health, mental health, and substance use in early adulthood: A cohort study of an urban, minority sample in the U.S. Child Abuse Negl. 2013, 37, 917-925. [CrossRef]

23. Miettunen, J.; Murray, G.K.; Jones, P.B.; Mäki, P.; Ebeling, H.; Taanila, A.; Joukamaa, M.; Savolainen, J.; Törmänen, S.; Järvelin, M.-R.; et al. Longitudinal associations between childhood and adulthood externalizing and internalizing psychopathology and adolescent substance use. Psychol. Med. 2014, 44, 1727-1738. [CrossRef]

24. Thiery, B.W.; Lange, S.; Rogelj, J.; Schleussner, C.-F.; Gudmundsson, L.; Seneviratne, S.I.; Andrijevic, M.; Frieler, K.; Emanuel, K.; Geiger, T.; et al. Intergenerational inequities in exposure to climate extremes. Science 2021, 374, 158-160. [CrossRef]

25. Burke, S.E.L.; Sanson, A.V.; van Hoorn, J. The psychological effects of climate change on children. Curr. Psychiatry Rep. 2018, 20, 35. [CrossRef] [PubMed]

26. Clemens, V.; von Hirschhausen, E.; Fegert, J.M. Report of the intergovernmental panel on climate change: Implications for the mental health policy of children and adolescents in Europe-A scoping review. Eur. Child Adolesc. Psychiatry 2020, 1-13. [CrossRef] [PubMed]

27. Bartlett, S. The implications of climate change for children in lower-income countries. Child. Youth Environ. 2008, 18, 71-98.

28. Helldén, D.; Andersson, C.; Nilsson, M.; Ebi, K.L.; Friberg, P.; Alfvén, T. Climate change and child health: A scoping review and an expanded conceptual framework. Lancet Planet. Health 2021, 5, e164-e175. [CrossRef]

29. Whiteford, H.A.; Ferrari, A.J.; Degenhardt, L.; Feigin, V.; Vos, T. The global burden of mental, neurological and substance use disorders: An analysis from the global burden of disease study 2010. PLoS ONE 2015, 10, e0116820. [CrossRef]

30. Grimshaw, J. A Guide to Knowledge Synthesis: A Knowledge Synthesis Chapter; Canadian Institutes for Health Research: Ottawa, ON, Canada, 2010; p. 56.

31. Tricco, A.C.; Lillie, E.; Zarin, W.; O’Brien, K.K.; Colquhoun, H.; Levac, D.; Moher, D.; Peters, M.D.J.; Horsley, T.; Weeks, L.; et al. PRISMA extension for scoping reviews (PRISMA-ScR): Checklist and explanation. Ann. Intern. Med. 2018, 169, 467-473. [CrossRef]

32. American Psychiatric Association Diagnostic and Statistical Manual of Mental Disorders (DSM-5®); American Psychiatric Pub: Washington, DC, USA, 2013; ISBN 978-0-89042-557-2.

33. World Bank. World Bank Country and Lending Groups. Available online: https://datahelpdesk.worldbank.org/knowledgebase/ articles /906519-world-bank-country-and-lending-groups (accessed on 5 November 2020).

34. Middleton, J.; Cunsolo, A.; Jones-Bitton, A.; Wright, C.J.; Harper, S.L. Indigenous mental health in a changing climate: A systematic scoping review of the global literature. Environ. Res. Lett. 2020, 15, 053001. [CrossRef]

35. Godin, K.; Stapleton, J.; Kirkpatrick, S.I.; Hanning, R.M.; Leatherdale, S.T. Applying systematic review search methods to the grey literature: A case study examining guidelines for school-based breakfast programs in Canada. Syst. Rev. 2015, 4, 138. [CrossRef] 
36. Sitwat, A.; Asad, S.; Yousaf, A. Psychopathology, psychiatric symptoms and their demographic correlates in female adolescents flood victims. J. Coll. Physicians Surg. 2015, 25, 886-890.

37. Peng, M.; Liu, A.; Zhou, J.; Wen, S.; Li, S.; Yang, T.; Li, X.; Huang, X.; Abuaku, B.; Tan, H. Association between posttraumatic stress disorder and preflood behavioral characteristics among children aged 7-15 years in Hunan, China. Med. Princ. Pract. Int. J. Kuwait Univ. Health Sci. Cent. 2011, 20, 336-340. [CrossRef]

38. Ahmad, N.; Bukhari, T.; Munir, N. The prevalence of post traumatic stress disorder (PTSD) among flood affected school children in Pakistan. Interdiscip. J. Contemp. Res. Bus. Inst. Interdiscip. Bus. Res. UK 2011, 3, 445-452.

39. An, Y.; Huang, J.; Chen, Y.; Deng, Z. Longitudinal cross-lagged relationships between posttraumatic stress disorder and depression in adolescents following the Yancheng tornado in China. Psychol. Trauma Theory Res. Pract. Policy 2019, 11, 760-766. [CrossRef] [PubMed]

40. Chowhan, A.; Margoob, M.A.; Mansoor, I.; Sakral, A. Psychiatric morbidity in children and adolescent survivors of a snowstorm disaster in South Kashmir, India. Br. J. Med. Pract. 2016, 9, a902.

41. Kar, N.; Mohapatra, P.K.; Nayak, K.C.; Pattanaik, P.; Swain, S.P.; Kar, H.C. Post-traumatic stress disorder in children and adolescents one year after a super-cyclone in Orissa, India: Exploring cross-cultural validity and vulnerability factors. BMC Psychiatry 2007, 7, 8. [CrossRef] [PubMed]

42. $\mathrm{Wu}, \mathrm{D} . ; \mathrm{Yin}, \mathrm{H} . ; \mathrm{Xu}, \mathrm{S}$.; Zhao, Y. Risk factors for posttraumatic stress reactions among Chinese students following exposure to a snowstorm disaster. BMC Public Health 2011, 11, 96. [CrossRef]

43. Hassan, F.U.; Singh, G.; Sekar, K. Children's reactions to flood disaster in Kashmir. Indian J. Psychol. Med. 2018, 40, 414-419. [CrossRef]

44. Quan, L.; Zhen, R.; Yao, B.; Zhou, X.; Yu, D. The role of perceived severity of disaster, rumination, and trait resilience in the relationship between rainstorm-related experiences and PTSD amongst Chinese adolescents following rainstorm disasters. Arch. Psychiatr. Nurs. 2017, 31, 507-515. [CrossRef]

45. Tavernier, R.; Fernandez, L.; Peters, R.K.; Adrien, T.V.; Conte, L.; Sinfield, E. Sleep problems and religious coping as possible mediators of the association between tropical storm exposure and psychological functioning among emerging adults in Dominica. Traumatology 2019, 25, 82-95. [CrossRef]

46. An, Y.; Yuan, G.; Liu, Z.; Zhou, Y.; Xu, W. Dispositional mindfulness mediates the relationships of parental attachment to posttraumatic stress disorder and academic burnout in adolescents following the Yancheng tornado. Eur. J. Psychotraumatol. 2018, 9, 1472989. [CrossRef]

47. An, Y.; Yuan, G.; Zhang, N.; Xu, W.; Liu, Z.; Zhou, F. Longitudinal cross-lagged relationships between mindfulness, posttraumatic stress symptoms, and posttraumatic growth in adolescents following the Yancheng tornado in China. Psychiatry Res. 2018, 266, 334-340. [CrossRef]

48. Li, X.; Huang, X.; Tan, H.; Liu, A.; Zhou, J.; Yang, T. A study on the relationship between posttraumatic stress disorder in flood victim parents and children in Hunan, China. Aust. N. Z. J. Psychiatry 2010, 44, 543-550. [CrossRef]

49. Xu, W.; Yuan, G.; Liu, Z.; Zhou, Y.; An, Y. Prevalence and predictors of PTSD and depression among adolescent victims of the summer 2016 tornado in Yancheng City. Arch. Psychiatr. Nurs. 2018, 32, 777-781. [CrossRef]

50. Xu, W.; Ding, X.; Goh, P.H.; An, Y. Dispositional mindfulness moderates the relationship between depression and posttraumatic growth in Chinese adolescents following a tornado. Personal. Individ. Differ. 2018, 127, 15-21. [CrossRef]

51. Xu, W.; Fu, G.; An, Y.; Ding, X.; Zhou, Y. Mindfulness, posttraumatic stress symptoms, depression, and social functioning impairment in Chinese adolescents following a tornado: Mediation of posttraumatic cognitive change. Psychiatry Res. 2018, 259, 345-349. [CrossRef]

52. Yuan, G.; Xu, W.; Liu, Z.; Liu, C.; Li, W.; An, Y. Dispositional mindfulness, posttraumatic stress disorder symptoms and academic burnout in chinese adolescents following a tornado: The role of mediation through regulatory emotional self-efficacy. J. Aggress. Maltreatment Trauma 2018, 27, 487-504. [CrossRef]

53. Yuan, G.; Xu, W.; Liu, Z.; An, Y. Resilience, posttraumatic stress symptoms, and posttraumatic growth in Chinese adolescents after a tornado: The role of mediation through perceived social support. J. Nerv. Ment. Dis. 2018, 206, 130-135. [CrossRef]

54. Zhang, Y.; Xu, W.; Yuan, G.; An, Y. The relationship between posttraumatic cognitive change, posttraumatic stress disorder, and posttraumatic growth among Chinese adolescents after the Yancheng tornado: The mediating effect of rumination. Front. Psychol. 2018, 9, 474. [CrossRef]

55. Zhen, R.; Quan, L.; Yao, B.; Zhou, X. Understanding the relationship between rainstorm-related experiences and PTSD among Chinese adolescents after rainstorm disaster: The roles of rumination and social support. Front. Psychol. 2016, 7, 1407. [CrossRef]

56. Mordeno, I.G.; Galela, D.S.; Nalipay, M.J.N.; Cue, M.P. Centrality of event and mental health outcomes in child and adolescent natural disaster survivors. Span. J. Psychol. 2018, 21, e61. [CrossRef]

57. Nalipay, M.J.N.; Mordeno, I.G. Positive metacognitions and meta-emotions as predictors of posttraumatic stress disorder and posttraumatic growth in survivors of a natural disaster. J. Loss Trauma 2018, 23, 381-394. [CrossRef]

58. Taukeni, S.; Chitiyo, G.; Chitiyo, M.; Asino, I.; Shipena, G. Post-traumatic stress disorder amongst children aged 8-18 affected by the 2011 northern-Namibia floods. Jamba Potchefstroom S. Afr. 2016, 8, 169. [CrossRef] [PubMed]

59. Zong, Y.; Chen, X. The 1998 flood on the Yangtze, China. Nat. Hazards 2000, 22, 165-184. [CrossRef]

60. Weiss, D.S.; Marmar, C.R. The impact of event scale-Revised. In Assessing Psychological Trauma and PTSD; The Guilford Press: New York, NY, USA, 1997; pp. 399-411. ISBN 978-1-57230-162-7. 
61. Lagmay, A.M.F.; Agaton, R.P.; Bahala, M.A.C.; Briones, J.B.L.T.; Cabacaba, K.M.C.; Caro, C.V.C.; Dasallas, L.L.; Gonzalo, L.A.L.; Ladiero, C.N.; Lapidez, J.P.; et al. Devastating storm surges of typhoon Haiyan. Int. J. Disaster Risk Reduct. 2015, 11, 1-12. [CrossRef]

62. Weathers, F.W.; Litz, B.T.; Keane, T.M.; Palmieri, P.A.; Marx, B.P.; Schnurr, P.P. The PTSD Checklist for DSM-5 (PCL-5). Available online: www.ptsd.va.gov (accessed on 13 February 2022).

63. Xu, Z.; Sheffield, P.E.; Hu, W.; Su, H.; Yu, W.; Qi, X.; Tong, S. Climate change and children's health-A call for research on what works to protect children. Int. J. Environ. Res. Public Health 2012, 9, 3298-3316. [CrossRef]

64. Mukherji, A.; Ganapati, N.; Rahill, G. Expecting the unexpected: Field research in post-disaster settings. Nat. Hazards 2014, 73, 805-828. [CrossRef]

65. Amstadter, A.B.; Acierno, R.; Richardson, L.K.; Kilpatrick, D.G.; Gros, D.F.; Gaboury, M.T.; Tran, T.L.; Trung, L.T.; Tam, N.T.; Tuan, T.; et al. Posttyphoon prevalence of posttraumatic stress disorder, major depressive disorder, panic disorder, and generalized anxiety disorder in a Vietnamese sample. J. Trauma. Stress 2009, 22, 180-188. [CrossRef]

66. Wind, T.R.; Joshi, P.C.; Kleber, R.J.; Komproe, I.H. The impact of recurrent disasters on mental health: A study on seasonal floods in northern India. Prehospital Disaster Med. 2013, 28, 279-285. [CrossRef]

67. Kar, N. Psychological impact of disasters on children: Review of assessment and interventions. World J. Pediatr. WJP 2009, 5, 5-11. [CrossRef] [PubMed]

68. Ali, G.-C.; Ryan, G.; Silva, M.J.D. Validated screening tools for common mental disorders in low and middle income countries: A systematic review. PLoS ONE 2016, 11, e0156939. [CrossRef]

69. Pfefferbaum, B.; Nitiéma, P.; Tucker, P.; Newman, E. Early child disaster mental health interventions: A review of the empirical evidence. Child Youth Care Forum 2017, 46, 621-642. [CrossRef]

70. Crabtree, A. Climate change and mental health following flood disasters in developing countries, a review of the epidemiological literature: What do we know, what is being recommended? Australas. J. Disaster Trauma Stud. 2012, 2012-1, 21-30.

71. Suldo, S.M.; Shaffer, E.J. Looking beyond psychopathology: The dual-factor model of mental health in youth. Sch. Psychol. Rev. 2008, 37, 52-68. [CrossRef]

72. Antaramian, S.P.; Huebner, E.S.; Hills, K.J.; Valois, R.F. A dual-factor model of mental health: Toward a more comprehensive understanding of youth functioning. Am. J. Orthopsychiatry 2011, 80, 462. [CrossRef] 